\title{
REMARQUES SUR LE DÉCHIFFREMENT DES MANUSCRITS MOZARABES ${ }^{1}$
}

\author{
Marie-Thérèse URVOY \\ Institut Catholique de Toulouse
}

Quiconque travaille sur des textes arabes chrétiens de l'occident commence par une remarque qui est un truisme: l'extrême pauvreté quantitative de ceux-ci. Si les recherches menées surtout depuis un demi-siècle ont élargi nos connaissances et donné un tableau assez varié de la production écrite mozarabe, on se heurte au fait qu'on n'en a pas moins, le plus souvent, affaire à des unica. Or, généralement, on utilise plusieurs sources manuscrites pour établir un texte et on ne travaille qu'exceptionnellement sur un document unique. Dans le domaine qui nous occupe, c'est l'inverse. Ce n'est guère que pour des passages de la Bible qu'on dispose d'une pluralité de documents comparables. Par suite on se trouve devant un dilemme. Doiton s'en tenir aux règles habituelles d'édition et se cantonner de ce fait à du matériel dont l'intérêt n'est pas capital? Ou doit-on s'attaquer à ce qui apporte une information plus pressante, quitte à ce que son expression soit moins sûre?

Pour le Psautier versifié ${ }^{2}$ on a conjointement les deux situations. La préface en prose est donnée par trois sources hétérogènes et permet une édition critique au sens habituel du terme. Les argumenta en prose placés en tête de chaque psaume ne sont plus donnés intégralement que par deux sources, la troisième n'en citant que quelques-uns et sous forme abrégée, ce qui donne des informations indirectes mais ne peut compter pour l'établissement du texte. Enfin la partie versifiée n'est, pour sa quasi-totalité, connue que par un unicum. Certes celui-ci est loin d'être totalement satisfaisant. Rien que pour le psaume 150, la citation qu'en fait le Lexique de Leyde $^{3}$ permet de rétablir trois mots sur seulement sept vers. Faut-il alors, comme l'a exprimé P. S. Van Koningsveld ${ }^{4}$,

\footnotetext{
${ }^{1}$ Par «manuscrits mozarabes» j'entends les textes rédigés en arabe par des chrétiens d'al-Andalus ou par leurs successeurs soit en territoires léonais et castillan, soit en déportation en Afrique du Nord. C'est à eux seuls que peut vraiment s'appliquer le terme de mozarabe, si celui-ci veut bien dire "qui se veut arabe" ( $c f$. Urvoy, D., "Les aspects symboliques du vocable 'Mozarabe'. Essai de réinterprétation", Studia Islamica 78 [1993], 117-153).

${ }^{2}$ Le Psautier mozarabe de Hafs le Goth, éd. et trad. Urvoy, M. Th., Toulouse, Presses Universitaires du Mirail, 1994.

${ }^{3}$ Van Koningsveld, P. S., "Psalm 150 of the Translation by Hafș al-Qūtī (fl. 889 A.D. [?]) in the Glossarium Latino-Arabicum of the Leyden University Library", Bibliotheca Orientalis XXIX , 5/6 (1972), 277-280.

${ }^{4}$ Au colloque de Casablanca en décembre 1992.
} 
attendre d'avoir recensé toutes les citations possibles afin de disposer d'une base documentaire assez variée? À cela on peut faire deux objections. Tout d'abord ces citations sont en tout petit nombre. P. S. Van Koningsveld en a publié, outre les sept versets du Ps. 150, trois qui sont tirées d'un document occidental ${ }^{5}$, mais non celles qu'il a trouvées dans des sources orientales et qui ne doivent pas être beaucoup plus nombreuses. Sur un volume d'environ quatre mille vers que représente l'ensemble du Psautier cela ne fait guère. Par ailleurs, on risque de se retrouver dans une situation comparable à celle que l'on a connu après avoir collationné les variantes du texte coranique: comment les exploiter? Quelle est la part, dans ces modifications, des fautes de mémoire ou de copie? N'y a-t-il pas eu contamination par des traditions parallèles? Etc. Toutes choses qu'on ne peut trancher que par la critique interne, ce qui nous ramène au point de départ.

On est donc obligé de mettre en sourdine les scrupules de l'érudition si on veut donner au public au moins une première version d'un document important ${ }^{6}$, quitte à ce que des découvertes ultérieures permettent d'améliorer le texte. Cela s'est produit ailleurs et semble admis par la majorité des chercheurs. Mais il est certain que cela ne résoud pas tous les problèmes, qui sont aussi de l'ordre de l'éthique personnelle. Dans son travail sur le Lexique de Leyde, P. S. Van Koningsveld lui-même a contesté l'édition Seybold du fait de ses déficiences, mais n'en a pas fait une édition critique, ce qu'a regretté F. Corriente ${ }^{7}$, sans pour autant préciser selon quelle méthode celle-ci serait possible à ses yeux. G. Levi della Vida, pour sa part, avait fait preuve de scrupules vis à vis du texte d'histoire universelle conservé (!) à la mosquée de Sidi 'Uqba à Kairouan, puisqu'il s'était contenté de publier, en 1962, une étude sur lui, et avait gardé par devers lui l'établissement du texte, réalisé dans des conditions très difficiles étant donné le très mauvais état du document, travail qui n'a été publié que par ses héritiers ${ }^{8}$.

Certes, la question du mauvais état de conservation des manuscrits est générale, mais elle est rendue plus aiguë dans le domaine qui nos occupe ici du fait du petit nombre de documents disponibles et du manque d'éléments de comparaison. D'où le danger des extrapolations hâtives sur la base de textes perçus superficiellement. N. Morata a fait remarquer malicieusement que F. Simonet lui-même n'y a pas

\footnotetext{
${ }^{5}$ Cf. ci-dessus n. 3, et "New Quotations from Hafṣ al-Qūtī translation on the Psalms", Bibliotheca Orientalis XXX (1973), 315.

${ }^{6}$ L'édition du Psautier versifié n'a été entreprise qu'à la demande expresse de Mgr Edelby qui souhaitait le voir paraître dans la collection Patrimoine arabe chrétien (ce qui a été empêché par divers événements). Mais son intérêt ne se limite pas à cet aspect "communautaire» et, par exemple, deux universitaires israéliens, J. Sadan et D. Wasserstein, on dit leur étonnement de ce qu'un texte de cette importance n'ait pas été édité plus tôt.

${ }^{7}$ Corriente, F., El léxico árabe estándar y andalusí del Glosario de Leiden, Madrid, 1991, p. 5.

${ }^{8}$ Levi della Vida, G., Note di Storia Letteraria Arabo-Ispanica, Rome, 1971, 123-191.
} 
échappé. Tout en faisant grand cas des notes marginales en arabe ajoutées à certain codex wisigothique, auxquelles il attribue une grande "érudition ecclésiastique», il s'est bien gardé de les traduire du fait de leur difficile lisibilité et "no transcribe sino el nombre Agustín y dos o tres palabras más»" ${ }^{9}$. Nous avons en effet affaire dans beaucoup de cas à des textes peu susceptibles d'être recopiés, soit à cause de leur support (gloses marginales de textes latins), soit à cause de leur audience très spécifique qui disparait avec la phagocytose des chrétiens par les musulmans (textes liturgiques et ecclésiastiques), soit enfin à cause de leur niveau intellectuel médiocre (comme le texte d'histoire universelle de Kairouan, qui n'intéresse que le chercheur moderne comme témoignage historique). À quoi il faut ajouter que nombre de documents ont pâti de la méfiance de l'Église romaine lors de la reconquista et que ceux qui n'ont pas été détruits comme suspects d'hérésie (notamment adoptianiste) ont été progressivement délaissés. J'ai noté, à propos d'un fragment d'une copie du grand Recueil de canons conciliaires, qu'il portait les traces de toutes les étapes de cet abandon: texte arabe normal, puis texte arabe vocalisé, ensuite résumé en latin en marge de ces derniers, et enfin grattage pur et simple en vue d'une réutilisation du parchemin ${ }^{10}$. Même pour un texte de plus grande audience comme le Psautier versifié, qui a servi aussi bien dans des communautés chrétiennes que juives, nous ne disposons que d'un exemplaire unique, copie du XVII siècle d'une version médiévale perdue - peut-être dans l'incendie de l'Escorial de 1671—, et elle-même non exempte de tares (trous, taches, écriture passée, voire effacée...).

Ces remarques générales étant faites, reste la question du déchiffrement proprement dit. On sait que les textes mozarabes sont en écriture maghrébine (marquée par une position particulière du point, suscrit pour le qăf, souscrit pour le $f \vec{a})$. Quant à la forme des lettres, G. Levi della Vida a parlé d'une graphie "typiquement mozarabe" ", mais il s'agit là plutôt d'une vue impressionniste et on ne dispose pas d'une description de ce qui pourrait être l'écriture mozarabe standard ${ }^{12}$.

\footnotetext{
${ }^{9}$ Morata, N., "Las notas árabes del Cod. \& I-14", Boletín de la Real Academia de la Historia LXXXVI (1925), 635-639.

${ }^{10}$ Urvoy, M. Th., "Note de philologie mozarabe», Arabica XXXVI (1989), 235-236.

${ }^{11}$ Note di Storia Letteraria..., p. 124.

${ }^{12}$ Faute d'un recueil paléographique mozarabe on trouvera des reproductions de manuscrits dans divers travaux dont voici une liste non exhaustive. Dodds, J. D. (éd.), Al-Andalus. The Art of Islamic Spain, New York, 1992, p. 174: document du IX s. De Bruyne, D. et Tisserant, E., "Une feuille arabo-latine de l'Epître aux Galates", Revue Biblique, N.S. VII (1910), 330, et une reproduction plus complète (4 p.) mais moins lisible dans Riosalido, J., "Nuevo estudio del manuscrito mozárabe de Siguienza", Boletín de la Asociación Española de Orientalistas XXIX (1993), 126-129: document d'env. 900. Goussen, H., Die Christlisch-arabischen Literatur der Mozaraber (Leipzig, 1909), passim: datations incertaines. Fleischer, H. L., «Beschreibung der von Prof. Dr. Tischendorf im J. 1853 aus dem Morgenlande zurückgebrachten christlisch-arabischen Handschriften", ZD.M.G. 8 (1854), 584-585; repris dans ses Kleinere Schriften III (1888), pl. III: copie d'env. 1100. Levi della Vida, G., Note..., 148-149: copie de la fin XIII ${ }^{\mathrm{e}}$ ou début XIV s.
} 
Le Cardinal Tisserant a donné des descriptions fines d'écritures du $\mathrm{IX}^{\mathrm{e}}$ siècle ${ }^{13}$, mais il reconnaissait également d'une part que l'écriture arabe ne permet pas de fixer «une date à peu près certaine ${ }^{14}$ comme le fait l'écriture latine, de l'autre que la graphie mozarabe a évolué parallèlement à celle du monde arabo-musulman ambiant, allant d'une "raideur et (...) simplicité du trait» initiales vers un "allure plus cursive» ${ }^{15}$. Il est vraisemblable, aussi, que l'histoire générale des Mozarabes a influé sur leur mode d'expression. Certains des textes les plus anciens, contemporains d'une plus grande liberté, sont, à quelques nuances près (essentiellement les tics graphiques maghrébins), d'une facture proche des productions chrétiennes orientales, notamment de celles émanant du monastère de $\mathrm{Mar} \mathrm{Saba}^{16}$. Au contraire, les documents plus récents, rédigés en des temps où les Mozarabes sont coupés de la chrétienté orientale, sont davantage comparables aux écrits musulmans maghrébins. Aussi P. S. Van Koningsvel a-t-il pu faire une première approche de la datation du Lexique de Leyde (vraisemblablement de la fin du XII ${ }^{\mathrm{e}}$ siècle) en le confrontant avec des manuscrits almoravides et almohades ${ }^{17}$.

Pas plus que -même s'il existe indéniablement une «allure d'ensemble» de la graphie mozarabe - il n'y a de modèle standard, il n'y a de règle unique de transcription de l'arabe. F. Simonet avait noté, sur les textes qu'il avait consultés, une large utilisation des semi-voyelles $w \bar{a} w$ et $y \bar{a}{ }^{18}$. Cette "orthographe typiquement vulgaire» (Levi della Vida) se retrouvera encore dans des copies tardives comme celle du texte d'histoire universelle de Kairouan ${ }^{19}$. Mais d'autres manuscrits, témoignant sans doute d'une plus grande arabisation de la part du scribe, suivent beaucoup moins cette voie ${ }^{20}$. Par contre on trouve plus fréquemment l'allongement de la fatha en alif (dans le Psautier versifié cela est fait systématiquement en fin d'hémistiche). R. Dozy relevait, pour sa part, dans le Lexique de Leyde, une tendance à "confond(re) les lettres de même organe, p. e.

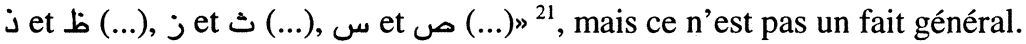

\footnotetext{
${ }^{13}$ De Bruyne, D. et Tisserant, E., «Une feuille...», 330; Tisserant, E., "Sur un manuscrit mozarabe de Fès", Miscellanea Biblica B. Ubach, Montserrat, (1953), 17.

${ }^{14}$ «Une feuille...", 324.

${ }^{15}$ Ibid., 330.

${ }^{16} \mathrm{Ibid}$. et "Sur un manuscrit mozarabe...", 17.

17 Van Koningsveld, P. S., The Latin-Arabic glossary of the Leiden Universitary Library. A contribution to the study of Mozarabic manuscripts and literature, Leyde, 1977, 25-31.

${ }^{18}$ Simonet, F., Glosario de voces ibéricas y latinas usadas entre los Mozárabes, nouv. éd., Amsterdam, 1967.

${ }^{19}$ Note..., p. 131.

${ }^{20}$ Cf. Urvoy, M. Th., "Note de philologie...”, 236.

${ }^{21}$ Dozy, R., Supplément aux dictionnaires arabes, $2^{e}$ éd., Leyde-Paris, 1927, I, IX.
} 
Cela nous conduit à nous interroger sur l'arabe employé par les Mozarabes. En fait il est impossible de donner une réponse globale, comme G. Troupeau a pu le faire pour l'orient chrétien, dont on dispose d'une production considérable ${ }^{22}$. Pour le Maghreb chrétien, les jugements sont aussi variés que les documents, dont le petit nombre, de surcroit, ne permet pas de dégager une tendance majoritaire. Dozy est frappé par les fautes contenues dans le Lexique de Leyde ${ }^{23}$. Par contre les passages attribuables au prêtre Vincentius, dans le Recueil de canons conciliaires, et publiés par Simonet ${ }^{24}$, semblent témoigner d'une bonne maîtrise de la langue ${ }^{25}$. Dans le texte d'histoire universelle qu'il a établi, Levi della Vida a remarqué à la fois son niveau linguistique et culturel très médiocre et son écriture «assez soignée, sans être élégante» ${ }^{26}$. Quant à $\mathrm{F}$. Taeschner, la traduction de l'Evangile sur laquelle il a travaillé lui a suggéré de chercher plutôt du côté de formes dialectales... ${ }^{27}$

Dans mon propre travail sur le Psautier versifié j'ai rencontré des phénomènes contradictoires qui m'ont laissée perplexe: le vocabulaire d'ensemble est plutôt réduit, et cependant surgit parfois un mot rare ${ }^{28}$; inversement, la grammaire est généralement correcte, et par moments on se heurte à des fautes grossières. Certaines s'expliquent par les besoins de la versification et peuvent passer pour des licences poétiques ${ }^{29}$, mais plusieurs ont des implications idéologiques qu'un arabe musulman ne pourrait tolérer, telle l'espèce de personnification d'actes divins, $d$ 'organes et de phénomènes psychologiques humains, voir d'animaux ${ }^{30}$.

La vocalisation, quand elle existe, pose des problèmes que seul un linguiste exercé pourrait peut-être résoudre. Tant dans le fragment du Recueil de canons concilaires que dans le Psautier versifié que j'ai manipulés, elle est extrêmement anarchique et souvent même contradictoire. Confronté lui aussi à un texte largement vocalisé, F. Taeschner a avoué sa perplexité car, tout en suggérant la piste du dialecte, il a ajouté

\footnotetext{
${ }^{22}$ Troupeau, G., Études sur le christianisme arabe au Moyen-Age, Aldershot, Variorum, 1995, I 5 , où il soutient le caractère classique - pour l'essentiel- de la langue arabe des chrétiens orientaux.

${ }^{23}$ Dozy, R., loc. cit.

${ }^{24}$ Simonet, F., Historia de los Mozárabes de España, reéd. Madrid, 1983, III, 720-724.

${ }^{25}$ Dans le texte même on a pu noter la présence d'un vocabulaire anté-islamique et coranique (AbuHaidar, J., "A Document of cultural Symbiosis: Arabic Ms. 1623 of the Escorial Library", Journal of the Royal Asiatic Society of Great Britain and Ireland (1987), 227.

${ }^{26}$ Note..., p. 124 et 131.

${ }^{27}$ Taeschner, F., "Die Monarchianischen Prologe zu den vier Evangelien in der spanisch-arabischen Bibelübersetzung des Isaak Velazquez nach der münchener Handschrift Cod. Arab. 238", Oriens Christianus 10 (1935), 81. Dans le Psautier versifié apparaissent plusieurs anomalies qui sont peut-être à rattacher à des dialectalismes (cf. Le Psautier mozarabe..., p. 79, 126, 133, 161, 164, 178 et 198). Dozy, Simonet et Levi della Vida ont également évoqué cette possibilité.

${ }^{28}$ Le Psautier mozarabe..., XIII.

${ }^{29}$ Ibid., p. 32, 66, 71, 73, 83, 118, 173, 211.

${ }^{30}$ Ibid., p. 42, 59, 73, 149.
} 
ne pas pouvoir continuer lui-même dans cette voie ${ }^{31}$. On pourrait, éventuellement, penser que.cette vocalisation a été ajoutée tardivement (c'est manifeste pour le fragment du Recueil de canons conciliaires), à une époque où la connaissance de la langue commençait à se perdre. Un spécialiste pourrait alors faire une "phenoménologie» de la désarabisation des Mozarabes. Mais ce n'est qu'une hypothèse.

Plusieurs textes mozarabes, notamment liturgiques, sont des traductions. Pour ce qui est de la transcription arabe de mots étrangers, il semble qu'il y ait eu une tendance prédominante mais non exclusive. Dans la version de l'Escorial du Recueil de canons conciliaires, les termes techniques (fonctions, grades ecclésiastiques...) sont transcrits dans une prononciation approchée mais systématique: gìm pour le c latin, $\underline{d} \bar{a} l$ pour le d, $\operatorname{s} \bar{\imath} n$ pour le s, $\underline{t} \vec{a}$ pour le $\mathrm{t}^{32}$. Par contre, le fragment du même texte conservé à Lisbonne est plus fluctuant. Le s est parfois transcrit par le $\sin$ et le d garde la sonorité explosive dāl en tête de mot, ne passant à la spirante $\underline{d} a \mathfrak{l}$ qu'en position intérieure. Quant aux lettres comme le

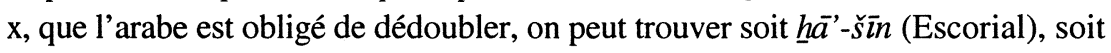
$\underline{h} \vec{a}$-gayn (Lisbonne) ${ }^{33}$. On retrouve ces mêmes problèmes pour la transcription des noms propres, notamment dans les Evangiles ${ }^{34}$.

Contrairement à ce qu'avait cru F. Simonet, le terme latin n'est pas toujours donné et on peut avoir d'emblée le terme arabe chrétien oriental. Il peut y avoir aussi des termes grecs. Si certains sont évidents, d'autres, même si parfois ils sont explicitement désignés comme tels, supposent la reconstitution d'étapes intermédiaires non attestées par ailleurs (ex.: diakihün, qui supposerait une forme comme diakeon, intermédiaire avec la forme classique diakonia). Enfin certaines transcriptions suggèrent la survenue d'une forte altération en passant du grec au latin puis du latin à l'arabe (ex.: alb(?)m, donné comme équivalent du latin tribunia, qui renverrait à $a m b o ̂ n)^{35}$.

Quant aux textes d'ensemble traduits ou adaptés du latin, la question qu'ils posent est double. D'une part il convient de déterminer exactement la source latine. De l'autre il s'agit de voir dans quelle mesure cette source permet de reconstituer le texte arabe quand le manuscrit est défectueux.

La difficulté qu'il y a à répondre à la première question est soulignée par le fait que même un texte bilingue peut être trompeur. Le fragment de l'Epître aux Galates

${ }^{31}$ Taeschner, F., loc. cit.

${ }^{32}$ Cf. Simonet, F., Glosario; Abu Haidar, J., “A document...”, p. 228; Kassis, H., "Arabic-Speaking Christians in al-Andalus in an Age of Turmoil (fifth/eleventh Century until A.H. 478/A.D. 1085)", $A l$ Qantara XV-2 (1994), 417.

${ }^{33}$ Urvoy, M. Th., "Note de philologie...".

${ }^{34}$ Taeschner, F., «Die monarchianischen Prologe...”, 81-83.

${ }^{35}$ Urvoy, M. Th., "Note de philologie...". 
de Sigüenza, par exemple ne donne pas un texte en traduction, mais deux traditions parallèles, une latine et une arabe $\mathrm{e}^{36}$. Outre la question de la plus grande liberté des premières traductions des Écritures, il y a des contraintes de l'adaptation, lesquelles sont plus particulièrement fortes dans le cas d'un texte poétique. Pour le Psautier versifié, P. S. van Koningsveld a opéré un sondage sur les psaumes 150 et 119-120 qui l'a fait conclure à une adaptation du Psalterium ex hebraico de Saint Jérôme ${ }^{37}$, c'est à dire la troisième et dernière édition faite par celui-ci de ce texte. La lecture de l'ensemble de l'oeuvre confirme cette hypothèse à deux réserves près. La plus importante, quantitativement parlant, est due au grand nombre d'ajouts faits par l'auteur mozarabe, que ce soit pour des raison de versification (chevilles de longueur variable), ou pour des besoins rhétoriques et doctrinaux (explicitation, exégèse, voire commentaires théologiques) ${ }^{38}$. On n'a donc là aucun élément de comparaison pour un volume qu'on peut estimer entre un cinquième et un quart des quelques quatre mille vers d'ensemble. La seconde réserve est que certains passages supposent que l'auteur mozarabe a travaillé soit sur plusieurs versions à la fois, soit —ce qui est le plus vraisemblable - sur une copie contenant des interpolations ${ }^{39}$.

Il est indéniable que le texte latin aide à lire le texte arabe en suggérant d'après le sens le mot qu'on ne peut déchiffrer ou dont on ne serait pas sûr autrement. Mais les réserves précédentes expliquent que, dans certains cas, la restitution n'est pas possible ${ }^{40}$. Quand, par contre, la traduction semble assez littérale, on peut remettre en place, par delà les effacements ou brouillages d'écritures, non seulement des mots mais des vers entiers ${ }^{41}$.

Toutefois, dans ce dernier cas, le texte latin ne permet pas, par une simple traduction littérale, de constituer un vers entier. Il faut alors "fabriquer une cheville». Ainsi, à la fin du Ps. 55, le passage effacé dans le manscrit Colville semble ne correspondre, chez Saint Jérôme, qu'à la fin de phrase suivante: "in luce viventium" (= finür ashạb al-hayr). C'est la rime avec le vers précédent qui suggère de rétablir le mot final $f a \bar{d} y^{\text {an }}$ (racheté), qui n'a pas d'équivalent dans le texte-source ${ }^{42}$. On procède ainsi un peu comme le public des séances de récitation poétique qui anticipe sur le mot-rime, et qui apprécie surtout l'auteur qui répond à leur attente.

On peut remarquer que, dans l'exemple donné, cela se fait assez naturellement: il suffit d'ajouter un mot simple à un fragment de phrase ordinaire. Même si la

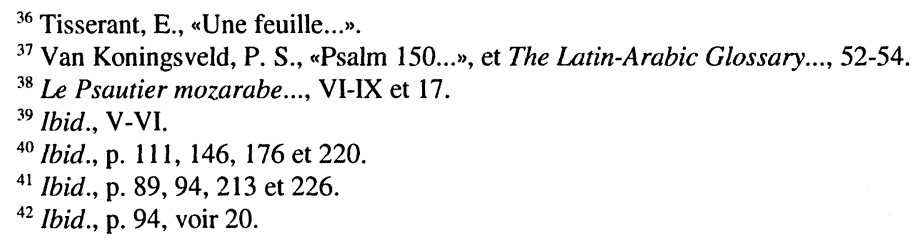


versification en rağaz utilisée ici est la plus simple, cela irait dans le sens de ce qui est dit par Ḥafṣ al-Qūtīi dans son urğūza introductive, à savoir que la restitution en langue sémitique du texte latin (lui-même établi d'après une confrontation scrupuleuse avec l'hébreu), coulerait de source ${ }^{43}$. Mais l'auteur reconnaît aussitôt que c'est loin d'être le cas pour tout le texte ${ }^{44}$, ce qui laisse subsister une grande part de difficulté. Ainsi, dans le Ps. 51, le verset "Sed Deus destruet te, in sempiternum, terrebit et evellet te de tabernaculo, et eradicavit te de terra viventium» est traduit par trois vers. Les deux premiers, dont le déchiffrement semble indiscutable, introduisent indubitablement des amplifications (soulignées ci-après):
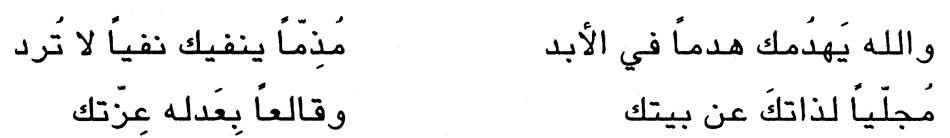

Dieu te ruinera, assurément, dans l'éternité; te blâmant ll t'exilera, certes, sans retour. Il te sortira de ta maison, dans Sa justice Il arrachera ton orgueil...

Quant au troisième vers, qui est illisible, on comprend qu'il exprime de terra viventium (min balad al-ahyā') et on sait que la rime doit être $k a$. Il faut donc se référer à la versification pour proposer la cheville nécessaire ( $k u l l^{i}$ așl $\left.{ }^{i} \ldots\right)$.

Ces remarques faites, il reste à espérer que l'avenir permettra la découverte d'autres textes afin d'enrichir notre connaissance d'une littérature arabe chrétienne certes marginale par rapport aux grandes productions de Mésopotamie d'abord, d'Egypt ensuite, mais qui n'en a pas moins le mérite d'exister et d'être le seul témoignage de ce genre dans l'islam d'occident.

\begin{abstract}
The reading of Christian Arabic manuscripts of al-Andalus has some specific aspects as a result of their small number, of their patterns of writting and of their connection with Latin texts.
\end{abstract}

\title{
RESUMEN
}

La lectura de los manuscritos árabes de al-Andalus presenta aspectos específicos por el hecho de su pequeño número, de su tipo de escritura y de sus vínculos con textos latinos.

${ }^{43} \mathrm{Ibid} .$, p. 16 , vers $49-55$.

${ }^{44}$ Ibid., vers 56. 